\title{
BMJ Open Implementation of a training program to increase knowledge, improve attitudes and reduce nursing care omissions towards patients with dementia in hospital settings: a mixed-method study protocol
}

\author{
Melina Evripidou, ${ }^{\circledR 1}$ Anastasios Merkouris, ${ }^{1}$ Andreas Charalambous, ${ }^{\oplus 1,2}$ \\ Evridiki Papastavrou ${ }^{1}$
}

To cite: Evripidou M, Merkouris A, Charalambous A, et al. Implementation of a training program to increase knowledge, improve attitudes and reduce nursing care omissions towards patients with dementia in hospital settings: a mixed-method study protocol. BMJ Open 2019;9:e030459. doi:10.1136/ bmjopen-2019-030459

- Prepublication history for this paper are available online. To view these files, please visit the journal online (http://dx.doi org/10.1136/bmjopen-2019030459).

Received 17 March 2019 Revised 16 June 2019 Accepted 28 June 2019

Check for updates

(C) Author(s) (or their employer(s)) 2019. Re-use permitted under CC BY-NC. No commercial re-use. See rights and permissions. Published by BMJ.

${ }^{1}$ Nursing, School of Health Sciences, Cyprus University of Technology, Limassol, Cyprus ${ }^{2}$ Nursing, Turun Yliopisto, Turku, Finland

Correspondence to Mrs Melina Evripidou; melinaevripidou@outlook.com

\section{ABSTRACT}

Introduction There is an evidence showing that when nurses have to allocate their time because of a lack of resources, older patients and especially those with dementia have a secondary care priority. The purpose of this study is to advance the level of knowledge, promote positive attitudes of nurses and reduce care deficits towards people with dementia through the implementation of a training programme. The programme will be enriched by an observational study of the care of patients with dementia to identify areas of missed care.

Method and analysis This study will follow a mixed methodology consisting of three stages: (1) evaluation of the level of nurses' knowledge and attitudes towards dementia care through the use of structured questionnaires, (2) observational study to evaluate nursing care in hospital settings, in order to detect any missed care and (3) quasi-experimental study, with a before-andafter design, through the implementation of the training programme in order to increase nurses' knowledge, improve attitudes and consequently to promote care for patients with dementia. The data will be analysed with descriptive and inferential statistics with the use of the SPSS V.24.0 and with content analysis as regard to the observational data.

Ethics and dissemination The protocol was approved by the National Bioethics committee and other local committees (EEBK: 2018.01.02). The participants will give their informed consent and the anonymity and confidentiality. Also, the protection of data will be respected. The results of the study will be disseminated in peer-reviewed international journals and conferences. If the intervention is successful, the training package will be given to the continuous education unit of the National Professional Association in order to be used on a regular basis.

\section{INTRODUCTION}

People with dementia (PwD) occupy 25\% of hospital beds ${ }^{1}$ and this number is expected to
Strengths and limitations of this study

- The main strength of the study lies in the fact that missed care, that is mainly measured through self-reporting instruments, will be detected through observation, increasing the innovation of the methodological approach of the problem.

- The educational intervention will not only focus on dementia knowledge deficiency, but it will also address specific areas of missed care for patients with dementia.

- The intervention will last for only 1 day and it is not certain that the effects will last for a long time, meaning that there will be a need to repeat educational interventions at a later stage.

- The study is going to be conducted only in three wards of the hospital, although nursing care settings are differential in other wards.

- During the second phase of the study, the total number of nurses per ward may be affected by sick leaves or training leaves and this might influence normal nursing care procedures.

triple by 2050, considering the rapid epidemiological increment of the disease. ${ }^{2}$ This group of patients is characterised by cognitive impairment placing them in an unfavourable position to demand the best possible care like other patients. ${ }^{3}$ Consequently, they might be in great danger of neglect. The increase in life expectancy associated with the multidimensional problem of dementia, inevitably leads to the need to investigate this topic. WHO $2017^{2}$ has highlighted the importance of the problem about dementia care in a global action plan on the public health response to dementia and set as a target $75 \%$ of countries to develop national policies, plans, policies 
either embedded with existing policies either independent until 2025. ${ }^{2}$

Studies have indicated that nurses have negative attitudes $^{4-6}$ and low level of knowledge towards dementia care. $^{7-10}$ This fact leads to the assumption that those patients are in danger of care omissions. Nurses have a key role for the improvement of quality of care as they are the ones who have the most contact and time with the patients. The acquisition of a high level of knowledge and positive attitudes will upgrade nursing ethics especially compassion, cognitive and emotional empathy for $\mathrm{PwD}$ among nurses, resulting in the promotion of quality of care. A significant part of WHO global plan is training health providers concerning the specials needs and management of care for PwD. For the accomplishment of this target, ${ }^{2}$ WHO recommends the development of training programmes for all heath professional and the encouragement to adopt positive attitudes. Promoting understanding and training will help to reinforce healthcare and improve social results, mainly quality of life for $\mathrm{PwD}$ and their carers, even the wider society leading to better living experience and settings for those people.

$\mathrm{PwD}$ are more vulnerable to care omissions than older people, as due to their cognitive impairment they cannot react or demand better quality of care. They are often marginalised and endangered from mistakes, omissions or even mistreatment, in relation with other groups of patients. This fact is attributed to the multidimensional nature, the memory and behaviour problems, the comorbidities and the complexity of care as well as with the associated management problems. As a vulnerable group of people, $\mathrm{PwD}$ are in danger for abuse or neglect. The concept of 'vulnerability' is defined as the reduced ability of an individual or group to predict, cope, resist and recover from the effects of natural or man-made risks. ${ }^{11}$ The concept is relative and dynamic in both directions.

During 2009, non-deliberate injury was the seventh cause associated with mistreatment of $65+$ people. $^{12}$ According to the ${ }^{13}$ the concept of 'mistreatment' in the elderly is defined as 'the only or repetitive act, or the lack of appropriate action, arising in any relationship where there is the expectation of confidence harm or risk to the elderly person.' Several studies have shown that the older people are at greater risk of neglect and the problem is more complicated by taking into account the level of comorbidity and potential risk. ${ }^{14-17} \mathrm{~A} \mathrm{study}^{18}$ has shown that chronic cases and the elderly, who had no apparent and immediate effects from the treatment, were often marginalised by health professionals. This focus on medical objectivity and impartiality is clearly rational and legitimate, but the consequences may be undesirable.

Highlighting the ethical perspective of missed nursing care, it seems that this phenomenon undermines the violation of fundamental human rights. People over the age of 65 with cognitive decline are often overlooked in their lived experienced and the preservation of human rights. ${ }^{3}$ In fact, people with 'silent minds' are deprived of their liberty by denying civil and political rights to society or even during hospitalisation. For PwD, more emphasis is given on providing basic care and supervision rather than integrating them into the wider community, so the human rights of those individuals are marginalised.$^{19}$ In addition, the political controversy over the human rights of $\mathrm{PwD}$ focuses on the right to respect and dignity, ${ }^{20}$ ignoring the fundamental rights of freedom and decision-making. ${ }^{19}$

In nursing, the topic of missed care, although it has been proven to exist since 2009, ${ }^{21}{ }^{22}$ is hardly acknowledged or discussed. The problem has recently brought up in the nursing scientific agenda through a European project ${ }^{23}$ (http://www.cost.eu/COST_Actions/ca/CA15208) that aims to bring together scholars, practitioners and stakeholders to develop a responsive research agenda that identifies challenges and innovative cost-effective and patient-centred solutions associated with missed care. The project will also enable research and policy synergies by drawing out the implications of missed nursing care across countries and identify innovative delivery models and strategies with an overall aim to address patient needs. This study is part of the project.

There are also some studies that brought evidence of care omissions and that nurses do not provide all the necessary care to patients. ${ }^{21-25}$ The main reasons for that phenomenon were lack of resources and time, so nurses prioritise care, leading to some processes to be delayed ${ }^{21}$ or undone, ${ }^{26}$ or not completed, ${ }^{27} 28$ or not delivered according to quality protocol standards. ${ }^{29}$ It has been demonstrated that missed nursing care is an international and important phenomenon, which might take place more frequently, due to the fact that nurses are in continuous interactions with patients and they are called to response in the whole spectrum of patients' needs, in an extremely complex environment, taking multiple tasks at the same time. Although in the context of care impartiality is necessary, in exceptional circumstances a patient might be prioritised. ${ }^{30}$

Two systematic reviews on missed nursing care ${ }^{27} 31$ have shown that communication, dental health, education, documentation, mobilisation, supervision, update of care plan, personal care, the medication, preparation for discharge ${ }^{2732-38}$ and psychological support ${ }^{26}$ were the most frequent omissions by nurses. These studies have not indicated if missed nursing care was correlated with a specific group of patients. A study ${ }^{39}$ revealed that nurses prioritise care in the basis of age or mental impairment of the patient, resulting in discrimination of care for older people, as they come last, during prioritisation process. Many studies have associated the quality of care and missed nursing care. $.^{18}{ }^{35} 40-43$ In addition patient safety, falls, immobility, dyspnoea, incontinence, ${ }^{22} 414445$ pneumonia, urine infection, delirium ${ }^{252646}$ and in hospital infections ${ }^{26} 4145$ have been proven as an impact of the phenomenon on patients.

A systematic review ${ }^{47}$ has identified the reasons for the low level of knowledge and negative attitudes and the beneficial action of intervention programmes to nurses. The main objectives of the study are to reveal the level 
of knowledge and attitudes of nurses towards $\mathrm{PwD}$ and to detect if any nursing care is missed. Last objective is the establishment of a qualified programme, which will improve the quality of care, provided to PwD.

\section{METHOD AND ANALYSIS \\ Aim}

This study aims to advance the level of knowledge, promote positive attitudes of nurses and reduce care deficits towards PwD through the implementation of a training programme. Specifically the aims are:

1. To explore the level of knowledge and nurses' attitudes towards the care of PwD.

2. To explore the care and care deficits of $\mathrm{PwD}$, aiming to get more information about nurses' knowledge and attitudes, so as to strengthen the training programme.

3. To implement a training programme, in order to increase knowledge, improve attitudes and reduce missed care for patients with dementia.

\section{Design}

This study is a mixed-method study, consisting of three stages. First, a descriptive study will take place, to evaluate the level of knowledge and attitudes of all acute hospital nurses, through the use of structured questionnaires. Second, an observation study will be held in order to detect the kind of care that is provided in hospital settings towards PwD. Lastly, a quasi-experimental study will follow with one group of nurses, including one pretest and two post-tests. This will be accomplished by the implementation of a 1-day training programme to one group of acute hospital nurses. The group will consist of 25 nurses and the intervention will be specifically designed for increasing the knowledge and attitudes of nurses towards $\mathrm{PwD}$. The reasons that quasi-experimental design was selected are due to the fact that there will not be a control group, and the participants cannot be randomly selected because they will need to participate in their free time.

\section{Stage 1: evaluation of the level of knowledge and the attitudes of nurses towards dementia care}

Through a systematic review of the literature, regarding knowledge and attitudes of nurses towards dementia care it has been revealed that knowledge was lacking and attitudes were non-favourable. ${ }^{47}$ So, there is a possibility that the quality of dementia care is affected. In this stage, two structured questionnaires will be used: (1) the Dementia Knowledge Assessment Tool V.2 (DKAT) ${ }^{48}$ for knowledge and (2) the Dementia Attitudes Scale, ${ }^{49}$ as far as it concerns attitudes. Both instruments were used in previous studies supporting their validity and reliability. ${ }^{48-51}$ The sample will include all nurses, working in acute hospital settings, specifically in medical, surgical and orthopaedic departments, of the five main general hospitals of the country. The specific hospital departments were selected, due to the high accommodation frequency of $\mathrm{PwD} .{ }^{20}$ Power analysis revealed sample estimation at 364 participants.
Approximately 400 questionnaires are planned to be distributed and the response rate must be over $70 \%$. Paediatric wards, outpatient clinic, emergency and maternity department will be excluded as they do not provide care to those patients, as frequently as others wards. Inclusion criteria are:

- Registered nurses based on the national law.

- Working in medical, surgical and orthopaedic department of the hospital for at least 1 year.

- Voluntary participation in study.

Questionnaires will be completed anonymously and the aim of the study will be explained in the first page prior to the questionnaires, along with the details for the contact person for further details. The completion of the questionnaires will be considered as the consent of participation. Questionnaires will be distributed by the researcher and returned to a closed box at the office of each ward in an envelope.

\section{Dementia Knowledge Assessment Tool V.2}

The first part of both questionnaires will include demographic information. The DKAT ${ }^{48}$ is used to measure the knowledge of nurses and caregivers concerning dementia and was designed exclusively for these groups. ${ }^{4}$ It contains 21 correct/wrong/ not know answers for assessing knowledge regarding causes, risk factors, disease symptomatology, care, impact, evaluation and management. The correct answers range from 0 to 21, with the highest score indicating more knowledge. It has been used by other researchers ${ }^{48}$ and it is a valid and reliable tool (Cronbach's alpha $=0.79){ }^{48}$

\section{Dementia Attitude Scale}

The Dementia Attitude Scale (DAS) ${ }^{49}$ was designed to investigate nurses' attitudes towards $\mathrm{PwD}$. It is divided into a seven-level Likert scale of 20 questions, which reflects the behavioural and cognitive components of nursing attitudes towards PwD. ${ }^{5}$ It contains two factors (1) knowledge and (2) attitudes with accepted reliability (Cronbach's alpha $0.83-0.85)$ and validity $(r=0.96)^{49}$ in correlation with similar instruments. ${ }^{50}$ Probable scores range from 20 to 140 with the highest score indicating more positive attitude. ${ }^{51}$ Its development was based on the three-dimensional attitude model that defines three components: influence, behaviour and knowledge. ${ }^{52}$ It has been used in previous surveys ${ }^{49-51}$ and is a commonly accepted and reliable measurement tool.

For the data analysis, inferential analysis will be reported such as linear regression and parametric measures ( $\mathrm{t}$-test, analysis of variance, CIs and correlation coefficients Pearson) as needed and descriptive (mean, SD, median, frequencies). Sociodemographic correlates will be assessed in correlation with the level of knowledge and attitudes. Considering data analysis for the DKAT V.2, descriptive statistic will be used and multiple regression analysis. This analysis will be followed to control confounder factors that might influence variables. For DAS, non-parametric test and Wilcoxon signed-rank 
test will take place. All the analysis will be performed by SPSS V.22.0. Multiple imputations will take place in order to handle missing data.

Both the questionnaires will be translated and back translated according to the guidelines suggested by WHO, $2016^{53}$ and the validation will include a test-retest, Cronbach's alpha and a factor analysis. Translation process will include forward translation, then expert panel for backward translation, a pretesting and cognitive interviewing, until we reach the final version. This procedure will be done during the pilot phase of the study that will include a sample of 100 nurses working in the emergency departments across the country. Those 100 nurses will be included only for the pilot study and not for the main study. As nurses from the emergency department are not included in the main study, pilot study was selected to take place among this group of nurses. Furthermore, nurses working in emergency departments do not provide care to PwD that last more than 1 day, although PwD visit the particular department on frequent occasions. So, we concluded that it is interesting to investigate emergency department nurses' level of knowledge and attitudes, considering the frequent, but short-term visit of PwD to the department. For the first attempt of this intervention, we decided to include nurses from the general department since they have closer contact and care for longer periods for patients with dementia. We are planning to include emergency department nurses at a later stage.

\section{Stage 2: observation study to detect any missed nursing care towards PwD}

Data from the observational study will be collected through field notes, in order to investigate care provided to PwD. Based on the hypothesis that if nurses' knowledge is low and their attitudes are negative, there is the possibility that care is not up to the expected quality standards. These data are expected to give more information as to the care of $\mathrm{PwD}$ and especially care deficits, care omissions and missed care, aiming to strengthen the training programme and focus on specific issues that will facilitate the care delivered to this group of patients.

We have conducted a scoping review considering the methodology of observational study, so as to proceed with our design.$^{54-60}$ The observer will be the main researcher, who is a nurse. One week training and 1-week pilot study will precede the main study. The pilot study will include the determination and familiarisation with the environment, the data collection process and coordination with the observation timetable. ${ }^{60}$ This duration of time is expected to eliminate any factors that may influence nurses' behaviour, due to observer presence. Reliability of the data, on stage 2, can be doubted, as nurse will not perform normal procedures with an observer in the room, but we expect that after 2 weeks of observer stay they will feel comfortable and continue their everyday routine. This is the reason that data from the first 2 weeks are not going to be analysed.
The potential for nursing omissions towards $\mathrm{PwD}$ is a fairly sensitive issue that, if is explored through questionnaires, nurses may hide some information about what is actually done because of the fear of questioning their professionalism, so the observation method is expected to reflect a more objective picture of reality. Moreover is the only method that can collect data from real settings and the validity and objectivity cannot be doubted, since the data are collected by an external observer. Field notes will not include any characteristic of patient or nurses.

\section{Sample}

The sample will include PwD, who are hospitalised for any reason. The sample strategy in observational studies is purposeful sampling, since the particular research topic is investigated in a predetermined population. A patient will be observed for one shift daily (morning or evening). The night shift has been excluded, due to its uneven duration which is 12 hours compared with the 6 hours shift (morning-evening). The sample size will be determined by the data saturation criterion.

In qualitative methods, the sample is smaller and when the data renewal no longer exists, the collection process is considered adequate. ${ }^{61}{ }^{62}$ Inclusion criteria will be the diagnosis of dementia ${ }^{6364}$ and the hospitalisation of the person with dementia, regardless of the reasons. The researcher will be informed at the beginning of the shift by the departmental officer if $\mathrm{PwD}$ are hospitalised in the department. The researcher will ensure and manage confidentially the data and diagnosis and cannot use them for the purposes of any other research. In the case of more than one patient per ward, random sampling will be performed, and data will be collected for one patient at a time and then the patient will not be included again in the sample.

\section{Duration of observation}

The duration of the observational study will last 1 day per patient. If there is availability of patients the data collection process will last 10-12 days. In other case, the process will last until we reach 10 patients, with the maximum duration 1 month. ${ }^{545759606566}$

\section{Wards}

The departments that are going to be observed are medical, surgical and orthopaedic in one of the five selected hospitals. The specific sections of the hospital were selected on the basis of the Alzheimer's Society report, ${ }^{20} 2009$, which showed that $14 \%$ of patients with dementia were admitted to hospital after falls, $12 \%$ for hip replacement, $9 \%$ for urinary tract infection, $7 \%$ for respiratory infection and $7 \%$ due to stroke. In addition, another study ${ }^{67}$ added to the above-mentioned reasons dehydration, as well.

The observer will stay in the patients' room, not wearing a nurse uniform, preserving at least $3 \mathrm{~m}$ distance from the patients' bed ${ }^{6168}$ and record everything she sees in the context of care in her field notes. This distance is 
probably predetermined for respect patient's privacy. The observer will not participate in any intervention or error in order not to influence the result of the study and the normal process of the department, with only exception if is a life or death issue. Additionally, no communication will take place with nurses, patients or patients' relatives during the observers' stay. Each patient will be recorded in new field notes.

\section{Field notes}

Data collection in the observational study will be based on field notes written by the observer, during her stay in the patient's room. The notes will be 'methodological', 'descriptive' and 'theoretical'. Descriptive field notes will include information about anything the observer watches or hears, regarding communication among nursespatient, process and environment characteristics. ${ }^{69} 70$ Methodological field notes will be composed of the technique of data collection. In particular, those notes include the process that the observer will use to write down the data, for instance, the adjustment of the observer in the culture of the organisation. ${ }^{69}$ Theoretical field notes regard the conscious, controlled efforts of the researcher to come to the essence of her notes. Those notes are the personal interpretation of data by the observer. ${ }^{70}$ More specifically, the researcher tries to understand behaviours or processes and to document some ideas, questions, thoughts or concerns as a reflection of her data. Only the research team will be aware of the context of the field notes, so as not to influence the results of the study and to conserve the confidentiality and anonymity of the participants. Data will be coded and categorised, so content analysis will follow. As content analysis is a reflective process ${ }^{71}$ the identification and condensation of the meaning units, the coding and the categorisation into themes is going to be a repeated event, by at least two of the research team, in order to maintain the quality and the trustworthiness of the analysis. ${ }^{72}$ Themes will emerge through the data and no software package will be used.

\section{Stage 3: quasi-experimental study}

Once the descriptive and observation study have been completed, a quasi-experimental study, with a beforeand-after design, which will include one group and one pretest and two post-tests will follow. This part of the study will include the organising of a 1-day training course, which will facilitate nurses to provide a better and consistent care to $\mathrm{PwD}$. The training will consist of various topics and will be conducted by dementia experts in order to describe, the pathophysiology of the disease and the appropriate care needed for these patients. The study has no costs, as the experts are willing to participate in the course free of charge. Furthermore, no economic outcomes are expected to emerge through the particular stage of the study.

The systematic review of the literature ${ }^{47}$ considering intervention studies to nurses has detected eleven educational programmes ${ }^{52}$ 73-81 with a large variety of designs.
Most of them were seminars ${ }^{74-81}$ lasting from 7 hours, ${ }^{74} 76$ 1 day, ${ }^{781} 3$ days ${ }^{75}$ to 15 weeks. ${ }^{80}$ Other studies used 1-day workshops,${ }^{73}$ while another research team used a 1-day online seminar ${ }^{50}$ or educational video ${ }^{79}$ This fact could be attributed to the deficiency of an established and validated programme, so each research team created its own programme for the purpose of each research.

For the purpose of the present study, we have selected the 'Getting to Know me' programme (with the approval of the authors) ${ }^{76}$ as it has checked validity and reliability but also it has been applied in more than one studies ${ }^{76}$ and it has been adopted by the National Health Service. The creators suggest that the programme can be applied to other countries, as well, as the results were beneficial for acute hospital nurses. However, it is expected that after the observational study, some modifications will be necessary to be introduced in order to focus on minimising missed care. In particular, based on stages 1 and 2 results, the programme will be modified. For instance, if the level of nurses' knowledge seems to be low on stage 1, emphasis is going to be given on the particular topic. Regarding stage 2 results if missed care is detected in specifics aspects of care, for example, feeding, the training programme will focus on that. In general as far as it concerns missed care, if this is proven through stage 2 , a lecture focusing on this topic is going to be added in the training programme.

\section{Description of the programme}

This programme is specifically designed for acute hospital nurses that provide care to PwD. It includes lectures, video, a guide for communication skills, the form 'Getting to know me' and a 30-page book. The main topics of lectures are introduction to dementia, holistic care, communication, impact of hospital environment, person-centred care and management of aggressive behaviour. The programme is going to be translated to Greek and some modifications will take place, so as to respond to study sample's needs and to the findings derived from the observation study. The modification will be an addition of an experiential workshop and participants will have more interaction through role play. The programme will be implemented in 2 days and the sample will be twenty nurses per day, with a total number of 50 nurses.

As the programme includes many interaction exercises, sample size was premeditated according to creators' suggestions about the number of participants, which is estimated to be 20 nurses per day. ${ }^{76}$ Participant number in those studies is also suggested by literature to be maximum 40 people, divided in smaller groups. ${ }^{5} 50767880$ We concluded to include 25 nurses per day, so as to eliminate the risk of drop-out. In addition, we have conducted a power analysis and the estimated sample size was calculated to include 20 participants. Response rate should be higher than $95 \%$ for the reliability and validity of the study. To achieve the response rate reminder phone 
calls to participants will take place after the duration of 3 months, by the $\mathrm{PhD}$ candidate.

Inclusion criteria will be the same as the descriptive stage of the study. However, priority will be given to nurses who work most with PwD, such as medical, orthopaedic or surgical wards, since the descriptive study is going to take place among those departments. The setting of this phase is university's lecture hall and the target sample is 50 acute hospital nurses.

\section{Evaluations}

Three evaluations will include (1) prior to the implementation of the programme, (2) immediately after the intervention and (3) the last one will take place 3 months after the intervention ${ }^{74} 7581-84$ in order to see if the effect of the programme remains for a longer period. Data analysis will be the same as stage 1 , since the tools that will be used on stage one and three are the same. The time intervals have been selected on the basis of international bibliography from interventional studies, which also studied the knowledge and attitudes of nurses in the care of dementia, ${ }^{875}{ }^{81}$ or implemented other intervention programmes in nurses. ${ }^{82-84}$

Primary outcomes of this stage are (1) the evaluation of nurses' knowledge and attitudes which is expected to be advanced after the implementation of the programme and (2) the reduction of nursing omissions during nursing care. Secondary outcomes is the upgrade of the quality of care provided to $\mathrm{PwD}$, through the adoption of positive attitudes, (3) the reinforcement of sympathy among nurses to the particular group of patients and (4) the development of communication skills and management strategies on behalf of nurses, in order to reduce burden during nursing care of $\mathrm{PwD}$.

Participants are going to be informed about the scope of the training programme and their obligation to fill the three questionnaires before their registration to the training. The PhD candidate will be responsible for the information, registration and their consent to participate. The research team will be responsible for organising, enrolment and coordinating the training course and the participation of nurses will be voluntary. If more than 50 nurses are willing to take part random sampling will be followed. A certification will be given after the completion of the last questionnaires to those who participate, in order to increase interest for joining the training course.

Data from the three stages are going to be stored in the researcher's office, where no one but the researcher is allowed entry. Data monitoring will be performed by the supervising committee and the $\mathrm{PhD}$ candidate will be responsible for analysing, coding and writing the data. Furthermore, the committee will conduct range checks of the values as far as it concerns the questionnaires. Regarding the observational data, two members of the committee will separately proceed to the coding process and then a discussion will follow with the PhD candidate, in order to develop thematic analysis.
The translation process has been done during September to January 2019. The pilot study has started on 3 February and has ended on 29 April 2019, so data from the pilot study have already been collected. The present stage of the study is on stage 1 , the descriptive part. The questionnaires were distributed, since 10 May 2019, and this phase is expected to be finalised until 15-20 June. The first results are expected on 1 July 2019 from the descriptive stage and until 30 September 2019 the qualitative findings will be available. Data from the third stage are expected between 10-17 February 2020, since the intervention is programmed to be implemented on 19 September 2019 and the last data that are going to be collected are 3 months after the intervention. The timeline of the study is illustrated on figure 1.

\section{DISCUSSION}

Nursing care rationing can be considered as the direct indicator of quality of care deficiencies, with a clear pathway for patient outcomes and their experiences. In addition, is a key concept for early detection of problems, before major repercussions occur. Moreover, detecting nursing care rationing will result in an early recognition of a possible risk by nurses or policy-makers. ${ }^{85}$ The vulnerable group of patients with cognitive impairment is the best example of complexity in the elderly, but with the highest rates of complications occurring, during hospitalisation. Omissions are not just a complete or a single unfortunate event, but a series of mild forms of neglect.

Nursing care rationing is an international phenomenon, as many studies investigated the kind of care which is missed, ${ }^{2425} 2732$ 35-38 4586 factors that influence the phenomenon, ${ }^{21} 2640424387-91$ the consequences on patients $^{18} 2225-274042-4446$ 92-94 and on nurses. ${ }^{27} 4243$ 95-97 This study aims to add to the existing model of factors associated with nursing care rationing ${ }^{29}$ the factors of knowledge and attitudes, without marginalising the other factors already acknowledged (environmental, organisational, administrative). None of those studies have correlated nursing care rationing with a specific group of patients and since $\mathrm{PwD}$ constitute a major percentage of hospitalised patients, in correlation with the rapid epidemiological growth, the topic is of high importance. All in all, this is the first study that is going to recognise if there is any correlation among the low level of knowledge and quality of care and implement an educational programme, so as to promote care among PwD.

Although the reasons for care rationing have been investigated, this phenomenon is even observed in cases where the workload is not much. Nurses have become so familiar with the 'chaos' associated with unexpected events and breaks in their processes, so they continue to act with prioritisation even when they are not imposed by other factors. ${ }^{21} 98$ There have been reports of doctors and nurses placing older people at a lower priority, with a lot of interventions and medications, but little care and comfort. ${ }^{18}$ This event was described as spam, but it often 


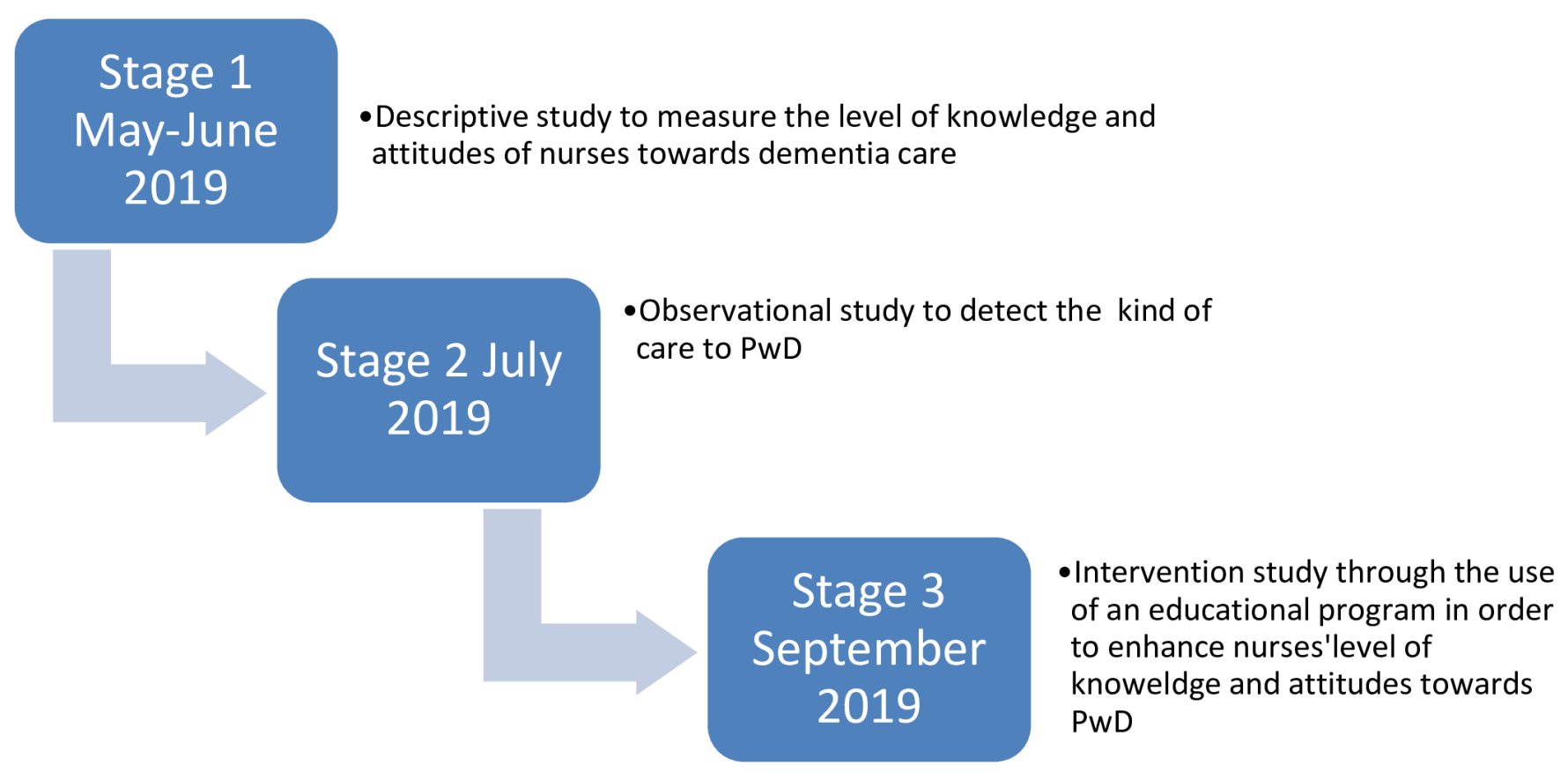

Figure 1 Timeline of the study. PwD, people with dementia.

happens. Prioritisation seems to be implemented through traditional biomedical approaches that go beyond ethical issues. $^{39}$

The study is going to be conducted only in three wards of the hospital, although nursing care settings are differential in other wards and this is a point for consideration. During the second phase of the study, the total number of nurses per ward may be affected by sick leaves or training leaves and this might influence normal nursing care procedures. The intervention will last for only 1 day and it is not certain that the effects will last for a long time, meaning that there will be a need to repeat educational interventions at a later stage. The generalisability of the study's results should be interpreted in the lights of these limitations.

\section{Patient and public involvement}

During the second stage of the study, patients and their carer participation are of high importance, by consenting to be observed. If they are not willing to do so, the study design for stage two will not be achievable. Research questions were informed based on published literature regarding carers' experience during hospitalisation of $\mathrm{PwD} .^{20}$ Also, informing them that care process is going to be observed they will share with us their priorities and their opinion about hospital care, so the outcome will be on stage three, as we will update our training programme based on patients' and carers' experience. During phases 1 and 2, patient and public involvement is not achievable, but stage 3 will be modified based on their experiences.

\section{ETHICS AND DISSEMINATION}

Access to the field of research was obtained by each hospital manager and the managers of each ward. The relatives of patients with dementia will be informed that patients will be observed and the aims of the study will be explained, so as to gain their permission.

After the observer will be informed about the rooms that hospice $\mathrm{PwD}$, she will be responsible for the recruitment of the patients and their carers. She will explain to both of them the aim, the design and the volunteer nature of the study, along with contact details for all the research team. If they agree, the relative will be asked to sign on the patients' behalf for participation in the study and then the data collection will start. Since PwD are characterised by cognitive impairment, their consent is often doubted. As patients' consent is an issue of conflict in the research field, ${ }^{99}$ we decided to proceed with the general practice regarding dementia studies and request relatives' signature ${ }^{100}$ regardless of patients' capacity. The patient will be informed despite of his/her capacity, which is not going to be assessed. Also, they will be informed that study results will be available in lectures that the researchers make in cooperation with the national Alzheimer Society. In cases that are not able to participate they can contact a member of the research team and inform them about the results of the study.

The anonymity, the trustworthiness and the process of the collected data will also be explained in the consent form both for the nurses and well as the relatives of PwD that the data are going to be used only for the purpose of the particular study and when is finished are going to be destroyed. In order to remain the confidentiality and 
the anonymity of the participants, none of the patients' or the nurses' identification will be included. Patients are going to be coded and no one except the main researcher will be able to know to whom the data are referred. The research team will be the only one who will have access to the data. If any important modification of the protocol will take place, all the relevant parties will be informed (ethics committee, health ministry). This study is in conjunction with the Standard Protocol Items: Recommendations for Interventional Trials. ${ }^{101}$

Dissemination strategy includes presentations in international and national scientific conferences. More emphasis will be given in nursing conferences, as the audience will be more benefited in clinical practice. All the results are going to be submitted in peer-reviewed journals for publication. Moreover, the results of the intervention stage are going to be presented in policy-makers in order for the programme to be implemented in a broader population of nurses. The results of the study are going to inform future study, as well, targeting carers of PwD and evaluate if the usefulness is as important as to nurses.

Acknowledgements The authors gratefully acknowledge nurses and departmental officers for their cooperation and the permission to conduct the observation study to their wards.

Contributors ME has written the manuscript. EP as the main supervisor of the project, contributed and supervised the writing process, reviewed the manuscript and consulted the first author. AM and AC have reviewed and contributed in writing the manuscript.

Funding The research is supported by Leventis foundation grant number EPI009/181120/820750. Publication fees were granted by the Cyprus University of Technology Open Access Author Fund.

Competing interests None declared.

Patient consent for publication Not required.

Ethics approval The protocol was approved by Cyprus National Bioethics committee (EEBK: 2018.01.02) and by the Research committee of the Ministry of Health.

Provenance and peer review Not commissioned; externally peer reviewed.

Open access This is an open access article distributed in accordance with the Creative Commons Attribution Non Commercial (CC BY-NC 4.0) license, which permits others to distribute, remix, adapt, build upon this work non-commercially, and license their derivative works on different terms, provided the original work is properly cited, appropriate credit is given, any changes made indicated, and the use is non-commercial. See: http://creativecommons.org/licenses/by-nc/4.0/.

\section{REFERENCES}

1. Alzheimer's Association. Alzheimer's disease facts and figures. 2016. Available at http://www.alz.org/documents_custom/2016Facts-and-Figures-Fact-Sheet.pdf (Accessed 19 Mar 2019).

2. WHO. Dementia: a public health priority. 2012. Available at http:// www.who.int/mental_health/publications/dementia_report_2012/en/ (Accessed 14 Feb 2019).

3. Knapp M. Economic outcomes and levers: impacts for individuals and society. Int Psychogeriatrc 2007;19:483-95.

4. Robinson A, Eccleston C, Annear M, et al. Who knows, who cares? Dementia knowledge among nurses, care workers, and family members of people living with dementia. J Palliat Care 2014;30:158-65.

5. Bryans M, Keady J, Turner S, et al. An exploratory survey into primary care nurses and dementia care. Br J Nurs 2003;12:1029-37.

6. Kada S, Nygaard HA, Mukesh BN, et al. Staff attitudes towards institutionalised dementia residents. J Clin Nurs 2009;18:2383-92.
7. Josefsson K, Sonde L, Wahlin TB. Competence development of registered nurses in municipal elderly care in Sweden: a questionnaire survey. Int J Nurs Stud 2008;45:428-41.

8. Galvin JE, Kuntemeier B, Al-Hammadi N, et al. "Dementia-friendly Hospitals. Alzheimer Disease Associated Disorders 2010;24:372-9.

9. Marx KA, Stanley IH, Van Haitsma K, et al. Knowing versus doing: education and training needs of staff in a chronic care hospital unit for individuals with dementia. J Gerontol Nurs 2014;40:26-34.

10. Barrett JJ, Haley WE, Harrell LE, et al. Knowledge about Alzheimer disease among primary care physicians, psychologists, nurses, and social workers. Alzheimer Dis Assoc Disord 1997;11:99-106.

11. International Federation if Red Cross and Red Crescent Societies (IFRC). What is vulnerability? 2014 http://www.ifrc.org/en/what-wedo/disaster-management/about-disasters/what-is-a-disaster/whatis-vulnerability/ (Accessed 21 Jan 2019).

12. Centers for Disease Control and Prevention (CDC). Elder Abuse. 2017 https://www.cdc.gov/violenceprevention/elderabuse/index. html (Accessed 3 Jan 2019).

13. WHO. Management of substance abuse. Process of translation and adaption of intruments. $2016 \mathrm{http} / / / \mathrm{www}$.who.int/substance_abuse/ research_tools/translation/en/ (Accessed 13 Mar 2019).

14. Dixon J, Manthorpe J, Biggs S, et al. Tinker A\&McCreadie C. Defining elder mistreatment: Reflections on the United Kingdom study of abuse and neglect of older people. Ageing Society 2010;30:403-20.

15. Gilhooly MLM, Cairns D, Davies M, et al. Framing the detection of financial elder abuse as bystander intervention: decision cues, pathways to detection and barriers to action. J Adult Protection 2013;15:54-68.

16. Manthorpe J, Abuse E. In: Dening. Oxford textbook of old age psychiatry. 2nd ed. Oxford: Oxford University Press, 2013:779-83.

17. Manthropre J. The abuse, neglect and mistreatment of older people with dementia in care home and hospitals in England: the potential for secondary data analysis: innovative practice. Dementia 2015;14:273-9.

18. Skirbekk H, Nortvedt P. Making a difference: A qualitative study on care and priority setting in health care. Health Care Analysis 2011;19:77-88.

19. Boyle M, Williams B, Tedd B, et al. Attitudes of undergraduate health science students towards patients with intellectual disability, substance abuse, and acute mental illness: a cross-sectional study. BMC Medical Education $2010 \mathrm{https}: / / \mathrm{bmcmededuc}$.biomedcentral. com/articles/ (Assessed 22 Feb 2019).

20. Alzheimer Society. Counting the cost. $2009 \mathrm{https} / / /$ www.alzheimers org.uk/download/downloads/id/787/counting_the_cost.pdf (Accessed 9 Feb 2019).

21. Kalisch BJ, Landstrom GL, Hinshaw AS. Missed nursing care: a concept analysis. J Adv Nurs 2009;65:1509-17.

22. Kalisch B, Tschannen D, Lee H F. Hospital variation in missed nursing care. Am J Med Qual 2012;26:291-9.

23. Scott PA, Harvey C, Felzmann $\mathrm{H}$, et al. Resource allocation and rationing in nursing care: A discussion paper. Nurs Ethics 2018:096973301875983.

24. Papastavrou $\mathrm{E}$. The ethics of care rationing within the current socioeconomic constraints. Health Science Journal 2012;6:362-4.

25. Ball JE, Griffiths P, Rafferty AM, et al. A cross-sectional study of 'care left undone' on nursing shifts in hospitals. J Adv Nurs 2016;72:2086-97.

26. Schubert M, Carke S, Glass T, et al. Identifying thresholdsfor relationship between impacts of rationing of nursing care and nurse and patient reported Outcomes in Swiss hospitals : A correlational study. Int J nurs Stud 2010;46:884-93.

27. Papastavrou E, Andreou P, Efstathioy G. Rationing of nursing care and nurses-patient outcomes: a systematic review of quantitative studies. Int J Health Manage 2014;2:3-25.

28. Verall C, Abery E, Harvey C, et al. Nurses and midwives perceptions of missed nursing care-A South Australian study. Collegian 2015;22(4):413-20.

29. Blackman I, Henderson J, Willis E, et al. Factors influencing why nursing care is missed. J Clin Nurs 2015;24(1-2):47-56.

30. Nortvedt P, Hem MH. The ethics of care: Role obligations and moderate partiality in health care. Nursing ethics 2011;18:192-200.

31. Jones T, Hamilton P, Murry N. Unfnished nursing care, missed care and implictly rationad care: state of the science review. Int $J$ of Nusing Studies 2015;52:1121-37.

32. Kalisch BJ, Xie B, Dabney BW. Patient-reported missed nursing care correlated with adverse events. Am J Med Qual 2014;29:415-22.

33. Kalisch BJ, Tschannen D, Lee KH. Missed nursing care, staffing, and patient falls. J NursCare Qual 2012;27:6-12. 
34. Lucero R. Nursing Informatics Implications from a Study of Unmet Nursing Care Needs. Connecting Health and Humans 2009;5043:817-21.

35. Aiken LH, Sloane D, et al. Nursing skill mix in European hospitals: cross-sectional study of the association with mortality, patient ratings, and quality of care. BMJ Qual Saf 2017;26:559-68.

36. Morin D, Leblanc N, money L. less care: how nurses in long-term care allocate hours of needed care in a context of chronic shortage. Int J Nurs Pract 2005;11:214-20.

37. Kalisch BJ, Xie B. Errors of omission: missed nursing care. Western J Nurs Res 2014;36:875-90.

38. Knopp-Sihota JA, Niehaus L, Squires JE, et al. Factors associated wth rushed and missed esident care in western Canada nursing homes: a cross-sectional survey of heath care aides. J Clin Nurs 2015;24:1815-25.

39. Pedersen R, Nortvedt $P$, Nordhaug $M$, et al. In quest of justice? Clinical prioritisation in healthcare for the aged. J Med Ethics 2008;34:230-5

40. Schubert M, Glass TR, Clarke SP, et al. Validation of the basel extent of rationing of nursing care instrument. Nurs Res 2007;55:416-24.

41. Sochalski J. Is more better?: the relationship be- tween nurse staffing and the quality of nursing care in hospitals. Med Care 2004;42(2):II-67-60.

42. Ausserhofer D, Schubert M, Desmedt M, et al. The association of patient safety climate and nurse-related organizational factors with selected patient outcomes: a cross-sectional survey. Int J Nurs Stud 2013;50:240-52.

43. Ausserhofer D, Zander B, Busse R, et al. Prevalence, patterns and predictors of nursing care left undone in European hospitals: results from the multicountry cross-sectional RN4CAST study. Br Med J Qual Safety 2014;23:126-35.

44. Lucero RJ, Lake ET, Aiken LH. Nursing care quality and adverse events in US hospitals. J Clin Nurs 2010;19(15-16):2185-95.

45. Hayward RA, Asch SM, Hogan MM, et al. Sins of omission: getting too little medical care may be the greatest threat to patient safety. $J$ Gen Intern Med 2005;20:686-91.

46. Brown P, Fraser $\mathrm{K}$, Wong CA, et al. Factors influencing intentions to stay and retention of nurse managers: a systematic review. J Nurs Manag 2012.

47. Evripidou M, Charalambous A, Middleton N, et al. Nurses' knowledge and attitudes about dementia care: systematic literature review. Pespectiv Psychiatr Care 2018.

48. Toye C, Lester L, Popescu A, et al. Dementia Knowledge Assessment Tool Version Two: development of a tool to inform preparation for care planning and delivery in families and care staff. Dementia 2014;13:248-56.

49. O'Connor M, McFadden S. Development and Psychometric Validation of Dementia Attitude Scale. Int $J$ of Alzheimer Disease 2010.

50. Kimzey M, Mastel-Smith B, Alfred D. The impact of educational experiences on nursing students' knowledge and attitudes toward people with Alzheimer's disease: A mixed method study. Nurse Educ Today 2016;46:57-63.

51. Scerri A, Scerri C. Nursing students' knowledge and attitudes towards dementia - a questionnaire survey. Nurse Educ Today 2013;33:962-8.

52. Breckler SJ. Empirical validation of affect, behavior, and cognition as distinct components of attitude. J Pers Soc Psychol 1984;47:1191-205.

53. WHO. Management of substance abuse. Process of translation and adaption of intruments. 2016http://www.who.int/substance abuse/ research_tools/translation/en/. (accessed 13 Sep 2018).

54. Välimäki T, Vehviläinen-Julkunen K, Pietilä AM. Diaries as research data in a study on family caregivers of people with Alzheimer's disease: methodological issues. J Adv Nurs 2007;59:68-76.

55. Ballermann MA, Shaw NT, Mayes DC, et al. Validation of the Work Observation Method By Activity Timing (WOMBAT) method of conducting time-motion observations in critical care settings: an observational study. BMC Med Inform DecisMak 2011:11:32.

56. Jackson D, Wilkes L, Luck L. Cues that predict violence in the hospital setting: findings from an observational study. Collegian 2014;21:65-70

57. D'Antonio S, Bagnasco A, Bonetti L, et al. Observation study on interruptions during nurses work in two surgical wards of a hospital in Liguria. Prof Inferm 2014;67:211-8.

58. Clisset $\mathrm{P}$, Porock $\mathrm{D}$, Harwood $\mathrm{RH}$, et al. The response of healthcare professionals to the admission of people with cognitive impairment to acute hospital setting: an observation and interview study. J Clin Nurs 2014;23(13-14):1820-9.
59. Hoviattalab K H. Cruz G, Halfens Ruud J G, Dassen T. Nursing practice in the prevention of pressure ulcers: An observational study of German Hospitals Nursing practice in the prevention of pressure ulcers: an observational study of German Hospitals. J Clin Nurs 2015:12:1513-24.

60. Tan Y, Wai T, Ng Sim C,I. Optimising peripheral venous catheter usage in the general inpatient ward: a prospective observational study. JClin Nurs 2016;26:133-9.

61. Cohen L, Manion L. Research methods in education. London, Routledge 2007.

62. Marshall M. Sampling for qualitative research. Family Practice 1996;13:5225.

63. Streit S, Limacher A, Zeller A, et al. Detecting dementia in patients with normal neuropsychological screening by Short Smell Test and Palmo-Mental Reflex Test: an observational study. BMC Geriatrics 2015;15:90

64. Travers C, Byrne GJ, Pachana NA, et al. Prospective observational study of dementia in older patients admitted to acute hospitals. Australas J Ageing 2014;33:55-8.

65. Hager K, Calabrese P, Frölich L, et al. An observational clinical study of the efficacy and tolerability of donepezil in the treatment of Alzheimer's disease. Dement Geriatr Cogn Disord 2003;15:189-98.

66. Goudra B, Singh PM, Galvin E. Comparison of compliance of glove use among anesthesia providers: a prospective blinded observational study. Aana J 2014;82:363-7.

67. Natalwala A, Potluri R, Uppal H, et al. Reasons for hospital admissions in dementia patients in Birmingham, UK, during 20022007. Dement Geriatr Cogn Disord 2008;26:499-505.

68. Creswell J, Vicki L, Plano C. Designing and conducting mixed method research. Los Angeles: SAGE Publications, 2011.

69. Bernard HR. Research methods in anthropology: Qualitative and quantitative approaches. Lanham, MD: AltaMira Press, 2011

70. Lonner WG, Berry JW. Field notes in cross-cultural research. Newbury Park.CA: Sage Publications, 1986.

71. Erlingsson C, Brysiewicz P. A hands-on guide to doing content analysis. Afr J Emerg Med 2017;7:93-9.

72. Bengtsson M. How to plan and perform a qualitative study using content analysis. NursingPlus Open 2016;2:8-14.

73. Kang Y, Moyle W, Venturato L. Korean nurses' attitudes towards older people with dementia in acute care settings. Int J Older People Nurs 2011:6:143-52.

74. Galvin JE, Kuntemeier B, Al-Hammadi N, et al. Dementiafriendly hospitals. Alzheimer Disease \& Associated Disorders 2010;24:372-9.

75. Surr CA, Smith SJ, Crossland J, et al. Impact of a person-centred dementia care training programme on hospital staff attitudes, role efficacy and perceptions of caring for people with dementia: A repeated measures study. Int J Nurs Stud 2016;53:144-51.

76. Elvish R, Burrow S, Cawley R, et al. 'Getting to Know Me': the development and evaluation of a training programme for enhancing skills in the care of people with dementia in general hospital settings. Aging Ment Health 2014;18:481-8.

77. Manthorpe J, Iliffe S, Eden A. Early recognition of dementia by nurses. J Adv Nurs 2003;44:183-91.

78. Davison TE, McCabe MP, Visser S, et al. Controlled trial of dementia training with a peer support group for aged care staff. Int J Geriatr Psychiatry 2007;22:868-73.

79. Einang Alnes R, Kirkevold M, Skovdahl K. Insights gained through Marte Meo counselling: experiences of nurses in dementia specific care units. Int J Older People Nurs 2011;6:123-32.

80. McCaffrey R, Tappen RM, Lichtstein DM, et al. Interprofessional education in community-based Alzheimer's disease diagnosis and treatment. J Interpro Care 2013;27:534-6.

81. Palmer JL, Lach HW, McGillick J, et al. The Dementia Friendly Hospital Initiative education program for acute care nurses and staff. J Contin Educ Nurs 2014;45:416-24.

82. Gustafsson M, Bohman DM, Borglin G. Challenges of conducting experimental studies within a clinical nursing context. App/Nurs Res 2014;27:133-6.

83. Borglin G, Gustafsson M. Krona H. A theory-based educational intervention targeting nurses' attitudes and knowledge concerning cancer-related pain management: a study protocol of a quasiexperimental design. BMC Health Serv Res 2011;23:233.

84. Mahedran R, Tan J, Griva K, et al. quasi-experimental, mixed methods investigation into the efficacy of a group psychotherapy intervention for caregivers of outpatients with cancer: the COPE study protocol. BMJ Open 2015;5:e008527.

85. Bail K, Berry H, Grealish L, et al. \&Peut A. Potentially preventable complications of urinary tract infections, pressure areas, pneumonia, and delirium in hospitalised dementia patients: Retrospective cohort study. BMJ Open 2013;3:6. 
86. Papastavrou E, Charalambous A, Vryonides S, et al. To what extent are patients' needs met on oncology units? The phenomenonofcarerationing. Eur J Oncol Nurs 2016;21:48-56.

87. Chassin MR, Galvin RW. The National Roundtable on Health Care Quality. The urgent need to improve health care quality: Institute of Medicine National Roundtable on Health Care Quality. J Am Med Assoc 1998;280:1000-5.

88. El-Jardali F, Lagace M. Making hospital care safer and better: the structure-process connection leading to adverse events. Healthc $Q$ 2005;8:40-8.

89. Halvorsen K, Slettebø A, Nortvedt P, et al. Priority dilemmas in dialysis - The impact of old age Priority dilemmas in dialysis : the impact of old age. J Med ehics 2008;34:585-9.

90. Tschannen D, Kalisch BJ, Lee KH. Missed nursing care: the impact on intention to leave and turnover. Can J Nurs Res 2010;42:22-39.

91. Castner J, YWB W, Dean-Baar S. Multi-level model of missed nursing care in the context of hospital merger. Western J Nurs Res 2014 (Accessed 11 Jan 2019).

92. Williams J. Care quality Commission Dignity and nutrition inspection program: National overview. 2011 https://www.cqc. org.uk/sites/default/files/documents/20111007_dignity_and_ nutrition_inspection_report_final_update.pdf (Accessed 1 Oct 2018).
93. McWhirter JP, Pennington CR. Incidence and recognition of malnutrition in hospital. BMJ 1994;308:945-8.

94. Rochefort CM, Clarke S. Nurses' work environments, care rationing, job outcomes, and quality of care on neonatal units. J Adv Nurs 2010;66:2213-24.

95. Dhaini SR, Zúñiga F, Ausserhofer D, et al. Care workers health in Swiss nursing homes and its association with psychosocial work environment: A cross-sectional study. Int J Nurs Stud 2016;53:105-15.

96. Vryonides S, Papastavrou E. \& Charalambous A. The ethical dimension of nursing care rationing : A thematic synthesis of qualitative studies. Nursing Ethics 2015;22:881-900.

97. Vryonides S, Papastavrou E, Charalambous A, et al. Ethical climate and missed nursing care in cancer care units. Nurs Ethics 2016;27.

98. Kalisch BJ. Missed nursing care: a qualitative study. J Nurs Care Qual 2006;21:306-13.

99. Howe E, Consent I. participation in research and the Alzheimer patient. Innov Clin Neurosci 2012;9:47-51.

100. Hedge S, Ellajisyula R. Capacity issues and decision-making in dementia. Ann Indian Acad Neurol 2016;19:34-9.

101. Chan A-W, Tetzlaff JM, Altman DG, et al. Statement: Defining standard protocol items for clinical trials. Ann Intern Med 2013;2013:200-7. 